\title{
Towards deterministic subspace identification for autonomous nonlinear systems
}

\author{
Alberto Padoan and Alessandro Astolfi
}

\begin{abstract}
The problem of identifying deterministic autonomous linear and nonlinear systems is studied. A specific version of the theory of deterministic subspace identification for discrete-time autonomous linear systems is developed in continuous time. By combining the subspace approach to linear identification and the differentialgeometric approach to nonlinear control systems, a novel identification framework for continuous-time autonomous nonlinear systems is developed.
\end{abstract}

Index Terms-Subspace identification, nonlinear identification, autonomous systems

\section{INTRODUCTION}

Building models of dynamical systems from observed measurements is an ubiquitous task in applications, particularly in model-based simulation, prediction and control of dynamical systems. In mathematical system theory this modelling task goes under the name of system identification. System identification methods are referred to as linear and nonlinear, depending on the model sought.

Prediction error methods and subspace identification methods broadly constitute the classical approaches to linear system identification [1-5]. The former revolves around the minimization of a prediction error criterion and is widely recognized as the state of the art for the identification of single-input single-output linear systems. The latter is coordinate-free and based on geometric arguments which draw upon linear realization theory. Subspace identification methods naturally deal with multi-input multi-output linear systems, and allow to identify such systems directly in statespace form [3-5]. By contrast, to identify multi-input multi-output linear systems, prediction error methods typically require the solution of computationally demanding non-convex optimisation problems [1,2]. In addition, subspace identification methods use tools of numerical linear algebra, which make them intrinsically reliable from a numerical point of view.

Subspace identification methods for discrete-time linear systems form nowadays a well-established field of research [3-5]. Different approaches have been used

A. Padoan is with the Department of Electrical and Electronic Engineering, Imperial College London, London SW7 2AZ, UK. (Email: alberto.padoan130imperial.ac.uk). A. Astolfi is with the Department of Electrical and Electronic Engineering, Imperial College London, London SW7 2AZ, UK and with the Dipartimento di Ingegneria Civile e Ingegneria Informatica, Università di Roma "Tor Vergata", Via del Politecnico 1, 00133, Rome, Italy. (Email: a.astolfi@imperial.ac.uk). to develop continuous-time counterparts of subspace identification methods [6-8]. However, the characterisation of their applicability to general nonlinear systems is still an open problem. A dedicated discrete-time subspace identification framework for linear parametervarying systems and for bilinear state-space systems has been developed in [9]. Subspace identification methods for discrete-time bilinear systems subject to white noise inputs have also been developed in [10,11]. Hammerstein-Wiener systems constitute another class of state-space nonlinear systems to which subspace identification methods have been extended [12-14]. Least squares support vector machines have been combined with the subspace identication approach to identify Hammerstein systems in [15]. Recently, subspace identification methods have been implemented in practical applications, particularly in the modelling of mechanical systems [16,17]. Finally, an interesting attempt to identify general nonlinear systems has been proposed in [18]. Therein, embedding techniques arising in the study of chaotic time-series have been combined with subspace identification methods. However, the initially adopted geometric approach is abandoned when neural networks are used to estimate the input-output mapping which describes the system to be identified. The reader is referred to $[19,20]$, and references therein, for in-depth discussions on the latest developments in nonlinear system identification.

The goal of this work is to propose a theoretical framework for the identification of deterministic continuous-time autonomous nonlinear systems. This framework combines the philosophy of subspace identification methods [3-5], which represent a conceptually simple and numerically reliable way to identify linear systems, and the differential geometric approach to nonlinear systems [21,22], which has significantly impacted the theory and applications of nonlinear control systems. Our strategy is to proceed as follows. A continuous time version of a specific subspace identification method for deterministic discrete-time autonomous linear systems is first derived. Then, a novel identification framework for continuous-time autonomous nonlinear systems is obtained by combining the subspace approach to linear identification and the differential-geometric approach to nonlinear systems.

The scope of this work is conceptual. The results concerning nonlinear systems boil down to the linear ones if specialized to the class of linear systems. Nonethe- 
less, the study of the nonlinear systems complements the study of the linear ones: while in the linear case it is often possible to provide stronger statements, certain phenomena or issues only occur in the nonlinear case, and do not have special interest (or meaning) in the linear one. This paper can be considered as a first preliminary step toward the development of a nonlinear enhancement of subspace identification methods.

The remainder of this paper is organized as follows. Section II defines essential notation. Section III contains the formulation of the continuous-time nonlinear identification problem for autonomous systems. In Section IV subspace identification methods for deterministic linear systems are developed in continuous-time. The main results are presented in Section V, where the differential geometric approach to nonlinear systems is combined with the modus operandi of subspace identification methods to solve the identification problem posed. Conclusions and future directions of research are given in Section VI.

\section{NotATION AND PRELIMINARIES}

The mathematical notation used is standard. $\mathbb{R}$, $\mathbb{R}^{n}$ and $\mathbb{R}^{p \times m}$ denote the set of real numbers, of $n$ dimensional vectors with real entries, and of $p \times m$ dimensional matrices with real entries, respectively. The symbol $A^{+}$is used to denote the Moore-Penrose inverse of the real matrix $A \in \mathbb{R}^{p \times j}$.

The projection of the row space of the matrix $A \in \mathbb{R}^{p \times j}$ on the row space of the matrix $B \in \mathbb{R}^{q \times j}$ is given by $A / B=A \Pi_{B}$, where the matrix $\Pi_{B} \in \mathbb{R}^{j \times j}$, defined as $\Pi_{B}=B^{\top}\left(B B^{\top}\right)^{\dagger} B$, denotes the projection matrix into the row space of the matrix $B$. The projection of the row space of the matrix $A \in \mathbb{R}^{p \times j}$ onto the orthogonal complement of the row space of the matrix $B \in \mathbb{R}^{q \times j}$ is given by $A / B^{\perp}=A \Pi \frac{\perp}{B}$, where the matrix $\Pi_{B}^{\perp} \in \mathbb{R}^{j \times j}$, defined as $\Pi_{B}^{\perp}=I-\Pi_{B}$, denotes the projection matrix into the orthogonal complement of the row space of the matrix $B$.

The singular value decomposition of a matrix $A \in \mathbb{R}^{i \times j}$ of rank $n>0$ is given by

$$
A=U \Sigma V^{\top}=U_{1} \Sigma_{1} V_{1}^{\top},
$$

where $U=\left[U_{1} U_{2}\right] \in \mathbb{R}^{i \times i}$ and $V=\left[V_{1} V_{2}\right] \in \mathbb{R}^{j \times j}$ are orthogonal matrices such that $U_{1} \in \mathbb{R}^{i \times n}, U_{2} \in$ $\mathbb{R}^{i \times(n-i)}, V_{1} \in \mathbb{R}^{j \times n}, V_{2} \in \mathbb{R}^{j \times(j-n)}$, and $\Sigma \in \mathbb{R}^{i \times j}$ is a block diagonal matrix of the form

$$
\Sigma=\left[\begin{array}{cc}
\Sigma_{1} & 0 \\
0 & 0
\end{array}\right] \in \mathbb{R}^{i \times j},
$$

with $\Sigma_{1} \in \mathbb{R}^{n \times n}$ a positive definite diagonal matrix. The elements on the main diagonal of $\Sigma$ are called the singular values of $A$.

The notation $y^{(k)}(t)$, with $k$ a positive integer, is used to denote the $k$-th order derivative of the function $y$, provided it exists.
The Lie derivative of the smooth function $h$ along the smooth vector field $f$ is defined as $L_{f} h=\frac{\partial h}{\partial x} f$, and the functions $L_{f}^{k} h$, with $k$ a non-negative integer, are defined recursively as $L_{f}^{k} h=L_{f}\left(L_{f}^{k-1} h\right)$, with $L_{f}^{0} h=$ $h$. If the smooth function $h$ is vector-valued, the Lie derivative is defined component-wise. The differential of a smooth mapping $\lambda$ is denoted by $d \lambda$.

\section{Problem formulation}

Consider a continuous-time, autonomous, nonlinear system described by equations of the form ${ }^{1}$

$$
\dot{x}=f(x), \quad y=h(x),
$$

in which $x(t) \in \mathbb{R}^{n}$ and $y(t) \in \mathbb{R}^{l}$ denote the unknown state and the measured output of the system, respectively. Assume, without loss of generality, that the state of the system evolves on an open set $\mathcal{X} \subseteq \mathbb{R}^{n}$ containing the (unknown) initial condition $x_{0} \in \mathbb{R}^{n}$ for all ${ }^{2} t \geq 0$.

The continuous-time nonlinear identification problem for autonomous systems is to determine the (unknown) dimension $n$ of the system (1) and the (unknown) system mappings $f: \mathcal{X} \rightarrow \mathcal{X}$ and $h: \mathcal{X} \rightarrow \mathbb{R}^{l}$, from a given finite sequence $\left\{y\left(t_{k}\right)\right\}_{k=0}^{M}$ of observed output measurements, with $\left\{t_{k}\right\}_{k=0}^{M}$ a strictly increasing finite sequence of time instants and $t_{0} \geq 0$.

Remark 1. A global solution to the identification problem posed above may not exist. While linear systems behave globally in the same way as they behave locally, it is not necessarily possible to establish global dynamical properties for most nonlinear systems. Without additional assumptions on the nonlinear system (1), the description of its dynamics may be determined only in a neighbourhood of the (unknown) initial condition. Our analysis has therefore a local nature. In other words, we restrict our goal to finding the behaviour of the system mappings only in a sufficiently small open neighbourhood containing the (unknown) initial condition.

Remark 2. There exist infinitely many nonlinear systems able to produce the output generated by system (1). More specifically, the system

$$
\dot{\chi}=f_{\tau}(\chi), \quad y=h_{\tau}(\chi),
$$

with mappings

$$
\begin{aligned}
& f_{\tau}(\chi)=\left[\frac{\partial \tau}{\partial x} f(x)\right]_{x=\tau^{-1}(\chi)}, \\
& h_{\tau}(\chi)=[h(x)]_{x=\tau^{-1}(\chi)},
\end{aligned}
$$

\footnotetext{
${ }^{1}$ The mappings $f$ and $h$ are assumed to be smooth, i.e. $C^{\infty}$.

${ }^{2}$ Similar considerations can be performed when the state of the system is only defined on a real interval of the form $\left[0, t_{\max }\right)$, with $t_{\max }>0$.
} 
and $\tau$ a (local) diffeomorphism ${ }^{3}$ defined on $\mathcal{X}$, has the same output behavior as the system (1). For this reason, the system mappings $f$ and $h$ cannot be uniquely identified from a set of output measurements without additional assumptions. To remove this shortcoming, any state-space realization of the system (1) of the form (2) is considered acceptable. In other words, the solution of the posed identification problem is only determined up to a (local) diffeomorphism.

Subspace identification methods make extensive use of structured matrices. Below, we define two structured matrices employed in the sequel. Let

$$
\begin{aligned}
Y_{i, j}(t) & =\left[\begin{array}{cccc}
y(t) & y^{(1)}(t) & \ldots & y^{(j-1)}(t) \\
y^{(1)}(t) & y^{(2)}(t) & \ldots & y^{(j)}(t) \\
\vdots & \vdots & \ddots & \vdots \\
y^{(i-1)}(t) & y^{(i)}(t) & \ldots & y^{(i+j-2)}(t)
\end{array}\right] \in \mathbb{R}^{l i \times j},(3) \\
X_{j}(t) & =\left[\begin{array}{llll}
x(t) & x^{(1)}(t) & \ldots & x^{(j-1)}(t)
\end{array}\right] \in \mathbb{R}^{n \times j},(4)
\end{aligned}
$$

denote the output matrix and the state matrix at time $t \geq 0$, respectively. Throughout the paper, the subscripts " $i$ " and " $j$ " represent the number of block rows and the number of columns, respectively. In addition, the time argument $t$ is considered to be a fixed nonnegative real number. The abbreviations $Y_{i, j}$ and $X_{j}$ are thus used, so that certain formulas are easier to read.

To solve the identification problem some assumptions on the system and the corresponding matrices are needed.

Let $\mathcal{O}$ denote the observation space of system (1), i.e. the linear space over $\mathbb{R}$ of functions on $\mathcal{X}$, defined as

$$
\mathcal{O}(x)=\operatorname{span}\left\{L_{f}^{k} h_{s}(x), s \in[1, l], k \geq 0\right\}, \quad x \in \mathcal{X} .
$$

The observation space $\mathcal{O}$ defines the observability codistribution

$$
d \mathcal{O}(x)=\operatorname{span}\{d \lambda(x), \lambda \in \mathcal{O}\}, x \in \mathcal{X} .
$$

System (1) is said to satisfy the observability rank condition at $x_{0} \in \mathcal{X}$ if $d \mathcal{O}\left(x_{0}\right)=n$ [22].

Assumption 1. The observability rank condition is satisfied for each $x \in \mathcal{X}$.

Assumption 1 guarantees that the system is locally observable at any point $x \in \mathcal{X}$. Assumption 1 can be regarded as a direct nonlinear counterpart of the standard observability assumption made in subspace identification, as it coincides with the linear notion of observability when the system is linear.

Assumption 2. The user-defined positive integers $i$ and $j$ are such that $n<i \leq j$.

Assumption 2 is needed to ensure that the system mappings can be (locally) determined. Typically, the positive integers $i$ and $j$ are such that $j$ is much larger

\footnotetext{
${ }^{3} \mathrm{~A}$ smooth bijection $\tau$ is a diffeomorphism if its inverse map $\tau^{-1}$ is also smooth.
}

than $i$ and such that all the available information is used. Note that this assumption requires an upper bound on $n$ to be known. In practice, since no algorithm is able to produce an upper bound of $n$, it is necessary to assume an upper bound a priori.

Assumption 3. The rank condition $\operatorname{rank}\left(X_{j}\right)=n$ holds for almost every ${ }^{4} t \geq 0$.

Assumption 3 can be interpreted as a controllability assumption. This condition implies that the columns of $X_{j}$ span the $n$-dimensional state space.

\section{Subspace identification for Autonomous LINEAR SYSTEMS}

In this section basic conventions and standard concepts of subspace identification for discrete-time autonomous linear systems are developed in continuoustime. From a theoretical point of view there are no significant differences between the discrete-time and the continuous-time linear frameworks. This is because discrete-time and continuous-time linear systems are described by formally identical matrix equations. Nonetheless, to motivate our approach and to highlight the peculiarities of the nonlinear case, it is instructive to illustrate a version of the continuous-time subspace identification framework for linear systems. With the exception of minor modifications, the approach proposed below is borrowed from [5] and adapted to the continuous-time scenario. Different subspace identification methods for continuous-time linear systems can be found in [6-8].

Consider a continuous-time, autonomous, linear system described by equations of the form

$$
\dot{x}=A x, \quad y=C x,
$$

in which $x(t) \in \mathbb{R}^{n}$ and $y(t) \in \mathbb{R}^{l}$, respectively. Autonomous linear systems can be regarded as a special class of systems of the form (1) in which the mappings $f$ and $h$ are linear functions of the state. For this class of systems, the output matrix $Y_{i, j}$ admits the decomposition

$$
Y_{i, j}=\Gamma_{i} X_{j}
$$

in which

$$
\Gamma_{i}=\left[\begin{array}{c}
C \\
C A \\
\vdots \\
C A^{i-1}
\end{array}\right] \in \mathbb{R}^{l i \times n},
$$

denotes the extended observability matrix and, without loss of generality, the state matrix can be written as

$$
X_{j}=\left[\begin{array}{llll}
x(t) & A x(t) & \ldots & A^{j-1} x(t)
\end{array}\right] .
$$

\footnotetext{
${ }^{4} \mathrm{~A}$ property is fulfilled for almost every $t \geq 0$, if the set where the property does not hold has a Lebesgue measure equal to zero.
} 
By Assumption 3 the matrix $X_{j}$ is full rank ${ }^{5}$. Thus, by observability of the system, equation (6) implies that the dimension of the system $n$ coincides with the rank of the output matrix $Y_{i, j}$, and, thus, that the column space of the extended observability matrix $\Gamma_{i}$ coincides with that of the output matrix $Y_{i, j}$. This fact allows to recover the dimension of the system $n$, and the column space of $\Gamma_{i}$ from the output matrix $Y_{i, j}$, which only requires measurements of the output $y(t)$ and its higher order derivatives. Consider the singular value decomposition

$$
Y_{i, j}=U_{1} \Sigma_{1} V_{1}^{\top}
$$

in which $\Sigma_{1} \in \mathbb{R}^{n \times n}$ is a full rank diagonal matrix. The dimension of the system $n$ is given by the number of non-zero singular values in the singular value decomposition above. In addition, the column space of the matrix $U_{1}$ coincides with that of the output matrix $Y_{i, j}$ and, hence, that of the extended observability matrix $\Gamma_{i}$. Thus, the relation

$$
U_{1}=\Gamma_{1} T=\left[\begin{array}{c}
C T \\
C T\left(T^{-1} A T\right) \\
\vdots \\
C T\left(T^{-1} A T\right)^{i-1}
\end{array}\right]=\left[\begin{array}{c}
C_{T} \\
C_{T} A_{T} \\
\vdots \\
C_{T} A_{T}^{i-1}
\end{array}\right]
$$

holds for some non-singular matrix $T \in \mathbb{R}^{n \times n}$ which represents an unknown similarity transformation. The matrix $C_{T}$ can be determined from the first $l$ rows of $U_{1}$. The matrix $A_{T}$ can be computed by solving the overdetermined linear system ${ }^{6}$

$$
\underline{U_{1}} A_{T}=\bar{U}_{1}
$$

which has a unique solution owing to Assumption 2. The original system matrices $A$ and $C$ are equivalent (up to a similarity transformation) to the estimated system matrices $A_{T}$ and $C_{T}$.

The construction of the output matrix $Y_{i, j}$ described above requires the evaluation of a large number of high-order time derivatives of the output signal $y(t)$. These are typically not available as measured data in most practical cases. For this reason, in continuous-time linear identification, specially designed filters are often used to filter the output signal and approximate their high-order time derivatives [24,25]. To alleviate this issue, the time invariance property can be exploited. More precisely, equation (6) also holds if the output

\footnotetext{
${ }^{5}$ Controllability is a generic property [23]: if a pair of matrices $(A, B) \in \mathbb{R}^{n \times n} \times \mathbb{R}^{n \times m}$ is considered as a point in a finitedimensional space, the set of controllable pairs is open and dense in the whole space. Thus, it is not restrictive to assume the controllabilty of the pair $(A, x(t))$, which, in turn, implies that the state matrix $X_{j}$ is full rank. Note also that if the pair $(A, x(0))$ is controllable, then the pair $(A, x(t))$ is controllable for all $t \geq 0$ finite.

${ }^{6}$ The symbols $\bar{U}_{1}$ and $U_{1}$ are used to denote $U_{1}$ without the first $l$ rows and the last $l$ rows, respectively.
}

matrix and the state matrix are defined as

$$
\begin{aligned}
Y_{i, j} & =\left[\begin{array}{cccc}
y\left(t_{0}\right) & y\left(t_{1}\right) & \ldots & y\left(t_{j-1}\right) \\
y^{(1)}\left(t_{0}\right) & y^{(1)}\left(t_{1}\right) & \ldots & y^{(1)}\left(t_{j-1}\right) \\
\vdots & \vdots & \ddots & \vdots \\
y^{(i-1)}\left(t_{0}\right) & y^{(i-1)}\left(t_{1}\right) & \ldots & y^{(i-1)}\left(t_{j-1}\right)
\end{array}\right] \in \mathbb{R}^{l i \times j}, \\
X_{j} & =\left[\begin{array}{cccc}
x\left(t_{0}\right) & x\left(t_{1}\right) & \ldots & x\left(t_{j-1}\right)
\end{array}\right] \in \mathbb{R}^{n \times j},
\end{aligned}
$$

where the time instants $\left\{t_{k}\right\}_{k=0}^{j-1}$ are all positive and distinct. With these modifications, in view of Assumption 2, the number of time derivatives of output measurements required for the construction of the output matrix $Y_{i, j}$ is drastically reduced.

Strategies similar to one described above have been combined with dedicated filtering techniques to design subspace identification algorithms for continuoustime linear systems in [6-8]. The approaches proposed in these works are undoubtedly suitable for practical implementation. However, at present, none of them has a clear and direct counterpart for nonlinear systems. By contrast, matrices of the form (3) and (4) allow to develop a subspace identification framework for nonlinear systems, as discussed in Section V. For this reason, we temporarily disregard the problem of approximating the required high-order time derivatives of the output signal. Throughout the paper, these quantities are assumed to be reliably obtainable from the observed finite sequence $\left\{y\left(t_{k}\right)\right\}_{k=0}^{M}$ of output measurements. An in-depth discussion of the issue of computing the derivatives of continuous-time variables which are known only through their time samples is given in [26]. The analysis of practical concerns, such as the effective sampling of the needed signals or the use of ancillary filtered versions of these signals, is the subject of ongoing research.

\section{SUbSPACE IDENTIFICATION FOR AUTONOMOUS NONLINEAR SYSTEMS}

As seen in the previous section, identifying autonomous linear systems boils down to solving a simple realization problem. By contrast, identifying nonlinear systems described by autonomous differential equations with output represents a nontrivial task.

Consider an autonomous nonlinear system with output described by equations of the form (1). To study the posed identification problem define the block Hankel matrix

$$
\mathcal{H}_{i, j}(x)=\left[\begin{array}{cccc}
h(x) & L_{f} h(x) & \ldots & L_{f}^{j-1} h(x) \\
L_{f} h(x) & L_{f}^{2} h(x) & \ldots & L_{f}^{j-2} h(x) \\
\vdots & \vdots & \ddots & \vdots \\
L_{f}^{i-1} h(x) & L_{f}^{i} h(x) & \ldots & L_{f}^{i+j-2} h(x)
\end{array}\right] \in \mathbb{R}^{l i \times j},
$$

for all $x \in \mathcal{X}$. If the matrix $\mathcal{H}_{i, j}$ is evaluated along the solutions of the system (1), one obtains the output matrix $Y_{i, j}$. Thus, the matrix $\mathcal{H}_{i, j}$ is assumed to be 
known at some fixed point $x \in \mathcal{X}$. Note that all the entries of the matrix $\mathcal{H}_{i, j}$ belong to the observation space of the system.

An important question now is whether a singular value decomposition of the matrix $\mathcal{H}_{i, j}(x)$, with $x \in \mathcal{X}$, allows to retrieve useful information about the system to be identified, just as in the linear case. Unfortunately, as explained below, the answer is negative for the vast majority of nonlinear systems. To illustrate this point, it is instructive to consider the case in which the observation space of system (1) is finite-dimensional.

\section{A. Preliminary discussion}

Suppose system (1) has a finite-dimensional observation space. A necessary and sufficient condition for a system to have a finite-dimensional observation space is to be immersed into a linear observable system ${ }^{7}$. By definition, this amounts to saying that there exists a (sufficiently) smooth mapping $\psi: \mathcal{X} \rightarrow \mathbb{R}^{N}$, satisfying $\psi(0)=0$, and real matrices $F \in \mathbb{R}^{N \times N}$ and $H \in \mathbb{R}^{l \times N}$, such that the pair $(F, H)$ is observable, which verify the conditions

$$
\frac{\partial \psi}{\partial x} f(x)=F \psi(x), \quad h(x)=H \psi(x) .
$$

Simple computations show that the conditions above imply that

$$
L_{f}^{k} h(x)=H F^{k} \psi(x)
$$

for each integer $k \geq 0$. Using this fact, it is immediate to verify that the matrix $\mathcal{H}_{i, j}(x)$, with $x \in \mathcal{X}$, admits the factorization

$$
\mathcal{H}_{i, j}=\Gamma_{i} X_{j}
$$

where

$$
\Gamma_{i}=\left[\begin{array}{c}
H \\
H F \\
\vdots \\
H F^{i-1}
\end{array}\right]
$$

and

$$
X_{j}=\left[\begin{array}{llll}
\psi(x) & F \psi(x) & \ldots & F^{j-1} \psi(x)
\end{array}\right]
$$

denote the extended observability matrix and the state matrix associated to the linear system

$$
\dot{\xi}=F \xi, \quad y=H \xi,
$$

in which the original system (1) is immersed, respectively. The well-defined restriction of the linear system (10) to the manifold described by $\xi=\psi(x)$, in turn, yields a copy of the dynamics of the nonlinear system (1). More precisely, the conditions (8) imply that the manifold $\mathcal{M}=\left\{(x, \xi) \in \mathcal{X} \times \mathbb{R}^{N}: \xi=\tau(x)\right\}$ is invariant under the flow of the augmented system

$$
\dot{x}=f(x), \quad \dot{\xi}=F \xi,
$$

\footnotetext{
${ }^{7}$ See [21, Chapter 8] for the definition of immersion of a system into another system and related results.
}

and that the output response produced by the system (1), when its initial state is $x_{0} \in \mathcal{X}$, can also be produced by the system (10) if the latter is set in the initial state $\psi\left(x_{0}\right) \in \mathbb{R}^{N}$. Equation (9) shows that the output matrix $\mathcal{H}_{i, j}$ decomposes into a linear combination of the rows of the state matrix $X_{j}$, determined by the extended observability matrix $\Gamma_{i}$. If the matrix $X_{j}$ is full rank $^{8}$, by observability of the pair $(F, H)$, equation (9) implies that the column space of $\Gamma_{i}$ coincides with that of $\mathcal{H}_{i, j}$. This implies that, in general, no information about the original system (1) can be retrieved by computing a singular value decomposition of the output matrix $Y_{i, j}$ when the observation space is finite-dimensional. By contrast, with this approach, one estimates the observability subspace of the linear system (10) in which the nonlinear system (1) is immersed. To illustrate this point consider the following academic example.

Example 1. Consider the nonlinear system described by equations of the form

$$
\dot{x}=f(x), \quad y=h(x),
$$

in which the state is $x(t)=\left(x_{1}(t), x_{2}(t), x_{3}(t)\right) \in \mathbb{R}^{3}$, the output is $y(t) \in \mathbb{R}$, the system mappings are defined as

$$
f(x)=\left[\begin{array}{lll}
x_{2}^{3} & x_{3} & 0
\end{array}\right]^{\top}, \quad h(x)=x_{1},
$$

for all $x \in \mathbb{R}^{3}$, and the initial condition of the state is $x(0)=x_{0} \in \mathbb{R}^{3} \backslash\{0\}$. The system satisfies the observability rank condition at any point of $\mathbb{R}^{3}$ except for the origin. Thus, the following considerations hold as long as the state of the system evolves away from the origin.

The nonlinear system (12) can be immersed in a five-dimensional observable linear system. To see this consider the mapping $\psi: \mathbb{R}^{3} \rightarrow \mathbb{R}^{5}$, defined as

$$
\psi(x)=\left[\begin{array}{lllll}
x_{1} & x_{2}^{3} & 3 x_{2}^{2} x_{3} & 6 x_{2} x_{3} & 6 x_{3}
\end{array}\right]^{\top},
$$

for each $x \in \mathbb{R}^{3}$. By defining the auxiliary variable $\xi=\psi(x)$, it is easy to see that the dynamics of the nonlinear system (12) can be described by a (nilpotent) linear realization of the form

$$
\dot{\xi}=F \xi, \quad y=H \xi,
$$

if the initial condition is set to $\xi(0)=\psi\left(x_{0}\right) \in \mathbb{R}^{5} \backslash\{0\}$, and the constant matrices $F \in \mathbb{R}^{5 \times 5}$ and $H \in \mathbb{R}^{1 \times 5}$ are chosen, for example, as

$$
\begin{aligned}
& F=\left[\begin{array}{lllll}
0 & 1 & 0 & 0 & 0 \\
0 & 0 & 1 & 0 & 0 \\
0 & 0 & 0 & 1 & 0 \\
0 & 0 & 0 & 0 & 1 \\
0 & 0 & 0 & 0 & 0
\end{array}\right], \\
& H=\left[\begin{array}{lllll}
1 & 0 & 0 & 0 & 0
\end{array}\right] .
\end{aligned}
$$

\footnotetext{
${ }^{8}$ Similarly to the linear case, assuming that the pair $(F, \psi(x))$ is controllable is not restrictive, because controllability is a generic property.
} 
This shows that, in a 5-dimensional space, the nonlinear system (12) can be seen as an observable nilpotent linear system. Note, however, that the linear realization (13) yields more output trajectories than the nonlinear realization (12). In other words, only certain initial conditions of the linear system (13) yield the state trajectories produced by the nonlinear system (12).

A direct consequence of the structure of system (12) is that by computing the singular value decomposition of the associated output matrix $Y_{i, j}$, one is only able to estimate the observability subspace of the (nilpotent) five-dimensional linear system in which it is immersed.

From the discussion above we conclude that the blind application of subspace identification methods developed for autonomous linear systems may prove inconclusive or even misleading when identifying autonomous nonlinear systems. Therefore, this task must be addressed using a different approach, which is discussed in the rest of this section.

\section{B. Main results}

To achieve the goal of identifying the autonomous nonlinear system (1) consider the following lemma.

Lemma 1. Let $\mathcal{X}$ be an open subset of $\mathbb{R}^{n}$. Let $h, \lambda$ be smooth functions and $f, \theta$ be smooth vector fields defined on $\mathcal{X}$. Define, recursively, the smooth functions $\rho_{k}^{\theta}$, with $k$ a positive integer, as

$$
\rho_{k}^{\theta}(x)=\sum_{q=0}^{k-1} L_{f}^{k-1-q} L_{[f, \theta]} L_{f}^{q} h(x),
$$

with $\rho_{1}^{\theta}(x)=L_{[f, \theta]} h(x)$, for all $x \in \mathcal{X}$. If

$$
\lambda=L_{\theta} h,
$$

then

$$
L_{f}^{k} \lambda=L_{\theta} L_{f}^{k} h+\rho_{k}^{\theta}
$$

with $k$ a positive integer.

Proof. The proof of this lemma follows directly from an induction argument and from the well-known identity [21, Chapter 1]

$$
L_{[f, \theta]} h=L_{f} L_{\theta} h-L_{\theta} L_{f} h .
$$

The property stated in Lemma 1 is instrumental to prove the followings result.

Theorem 1. Consider the nonlinear system (1). Assume that there exist smooth vector fields $\left\{\theta_{k}\right\}_{k=0}^{j-1}$ defined on $\mathcal{X}$ such that the condition

$$
L_{f}^{k} h=L_{\theta_{k}} h,
$$

is satisfied for each $k \in[0, j-1]$. Let

$$
\begin{aligned}
& \mathcal{H}_{i}(x)=\left[\begin{array}{c}
h(x) \\
L_{f} h(x) \\
\vdots \\
L_{f}^{i-1} h(x)
\end{array}\right] \in \mathbb{R}^{l i} \\
& \Theta_{j}(x)=\left[\begin{array}{llll}
\theta_{0}(x) & \theta_{1}(x) & \ldots & \theta_{j-1}(x)
\end{array}\right] \in \mathbb{R}^{n \times j}, \\
& R_{i, j}^{\theta_{0} \ldots \theta_{j-1}}(x)=\left[\begin{array}{cccc}
0 & 0 & \ldots & 0 \\
\rho_{1}^{\theta_{0}}(x) & \rho_{1}^{\theta_{1}}(x) & \ldots & \rho_{1}^{\theta_{j-1}}(x) \\
\vdots & \vdots & \ddots & \vdots \\
\rho_{i-1}^{\theta_{0}}(x) & \rho_{i-1}^{\theta_{1}}(x) & \ldots & \rho_{i-1}^{\theta_{j-1}}(x)
\end{array}\right] \in \mathbb{R}^{l i \times j},
\end{aligned}
$$

for each $x \in \mathcal{X}$. Then, the mapping $\mathcal{H}_{i, j}$, defined as in (7), admits the decomposition

$$
\mathcal{H}_{i, j}=d \mathcal{H}_{i} \Theta_{j}+R_{i, j}^{\theta_{0} \ldots \theta_{j-1}} .
$$

Proof. The claim is a direct consequence of Lemma 1.

Equation (16) establishes an important decomposition. It shows that the mapping $\mathcal{H}_{i, j}$ decomposes into a linear combination of the rows of the mapping $\Theta_{j}$, determined by the coefficient $d \mathcal{H}_{i}$, and an additional term $R_{i, j}^{\theta_{0} \ldots \theta_{j-1}}$. To simplify the notation, the superscripts " $\theta$ " and " $\theta_{0} \ldots \theta_{j-1}$ " representing the vector fields used to construct the mappings $\rho_{k}^{\theta}$ and $R_{i, j}^{\theta_{0} \ldots \theta_{j-1}}$ are dropped, if clear from the context, so that certain formulas are easier to read.

Before proceeding further with the analysis some additional assumptions on system (1) and the corresponding matrices are required.

Assumption 4. The output function $h$ of system (1) is linear, i.e. $h=C x$, for all $x \in \mathcal{X}$.

Assumption 4 guarantees that the output function $h$ is (locally) reconstructible from its differential $d h$. Note that, owing to the (local) observability assumption on the system, Assumption 4 is not restrictive.

Assumption 5. The intersection of the subspaces spanned by the rows of the matrices $\Theta_{j}(x)$ and $R_{i, j}(x)$ contains only the zero vector, for almost every $x \in \mathcal{X}$.

Note that the assumptions stated above are always verified when the system is linear.

Assumption 5 ensures that the following rank property holds.

Lemma 2. Consider system (1) and equation (16). Under the assumptions stated above, the rank condition

$$
\operatorname{rank}\left(d \mathcal{H}_{i} \Theta_{j}\right)=\operatorname{rank}\left(d \mathcal{H}_{i} \Theta_{j} / R_{i, j}^{\perp}\right),
$$

holds for each $x \in \mathcal{X}$.

Proof. The claim can be proved by applying elementary inequalities concerning the dimension of the intersection of linear spaces [27, Proposition 1.2]. 
The rank condition (17) allows to solve the identification problem posed. It implies that the the rank of the matrix $d \mathcal{H}_{i} \Theta_{j}$ does not change if it is projected on the row space of the matrix $R_{i, j}^{\perp}$.

The main advantage of introducing the decomposition (16) is as follows. Post-multiplying both sides of such equation by the projection matrix $\Pi_{R_{i, j}^{\perp}}$ yields

$$
\mathcal{H}_{i, j} / R_{i, j}^{\perp}=d \mathcal{H}_{i} \Theta_{j} / R_{i, j}^{\perp},
$$

where the identity $R_{i, j} \Pi_{R_{i, j}^{\perp}}=0$ has been used to eliminate the second term of the right-hand side. Assume for the moment that the matrix $\Theta_{j}$ is full rank, and recall that, by the observability assumption on the system, the matrix $d \mathcal{H}_{i}$ is full rank. Thus, by combining (17) and (18), we conclude that the rank of the matrix $\mathcal{H}_{i, j} / R_{i, j}^{\perp}$ is equal to the dimension of the system $n$, and, thus, its column space coincides with that of $d \mathcal{H}_{i}$. Note, however, that the matrix $\mathcal{H}_{i, j} / R_{i, j}^{\perp}$ does not require only measured data to be computed, as the matrix $R_{i, j}^{\perp}$ is unknown. Leaving aside the problem of determining the matrix $R_{i, j}^{\perp}$, which for the moment is assumed to be known, consider the singular value decomposition

$$
\mathcal{H}_{i, j} / R_{i, j}^{\perp}=U_{1} \Sigma_{1} V_{1}^{\top},
$$

in which $\Sigma_{1} \in \mathbb{R}^{n \times n}$ is a full rank diagonal matrix. The dimension of the system $n$ is given by the number of non-zero singular values in the singular value decomposition above. In addition, the column space of the matrix $U_{1}$ coincides with that of the output matrix $\mathcal{H}_{i, j} / R_{i, j}^{\perp}$ and, hence, that of the matrix $d \mathcal{H}_{i}$. Thus, the relation $U_{1}=d \mathcal{H}_{i} T$ holds for some non-singular matrix $T \in \mathbb{R}^{n \times n}$ which represents the inverse of the Jacobian matrix of an unknown (local) diffeomorphism. More precisely, if we denote by $\tau$ such (local) diffeomorphism and let $T=\frac{\partial \tau^{-1}}{\partial \chi}$, the relation above can be rewritten as

$$
U_{1}=d \mathcal{H}_{i} \frac{\partial \tau^{-1}}{\partial \chi}=\left[\begin{array}{c}
d h \\
d L_{f} h \\
\vdots \\
d L_{f}^{i-1} h
\end{array}\right] \frac{\partial \tau^{-1}}{\partial \chi}=\left[\begin{array}{c}
d h_{\tau} \\
d L_{f_{\tau}} h_{\tau} \\
\vdots \\
d L_{f_{\tau}}^{i-1} h_{\tau}
\end{array}\right]
$$

The function $h_{\tau}$ can be determined from the first $l$ rows of $U_{1}$. The mapping $f_{\tau}$ can be computed (at the point $x \in \mathcal{X}$ in which the matrix $\mathcal{H}_{i, j} / R_{i, j}^{\perp}$ is evaluated) by solving the overdetermined linear system

$$
\underline{U_{1}} f_{\tau}=\bar{U}_{1},
$$

which has a unique solution owing to Assumption 2. The original system mappings $f$ and $h$ are equivalent (up to a local diffeomorphism) to the obtained system mappings $f_{\tau}$ and $h_{\tau}$. In general, these mappings are only a local solution of the identification problem posed.

\section{A special case}

Consider now the question of finding a full rank matrix $\Theta_{j}$, the columns of which satisfy the condition (15). Consider the system (1) and define recursively the vector fields $\theta_{k}$, with $k$ a non-negative integer, as

$$
\theta_{k}(x)=\frac{\partial \theta_{k-1}}{\partial x} f(x)
$$

with $\theta_{0}(x)=x$, for all $x \in \mathcal{X}$. Observe that $\theta_{k}(x(t))=x^{(k)}(t)$, for all $t \geq 0$. Along the solutions of the system (1), the vector fields defined above coincide with the state of the system $x(t)$ and its successive time derivatives, and, thus, the matrix $\Theta_{j}$ coincides with the state matrix $X_{j}$. Under the stated assumptions, any vector field $\theta_{k}$ defined as in (19), with $k$ a non-negative integer, satisfies the condition (15) and the matrix $\Theta_{j}$ must be full rank for almost every $x \in \mathcal{X}$.

\section{On the matrix $R_{i, j}^{\perp}$}

We complete this section discussing the issue of determining the matrix $\mathcal{H}_{i, j} / R_{i, j}^{\perp}$, since in general the matrix $R_{i, j}^{\perp}$ is unknown. Although the remainder term $R_{i, j}$ cannot be distinguished from the product $d \mathcal{H}_{i} \Theta_{j}$ because only the output matrix $Y_{i, j}$ (i.e. $\mathcal{H}_{i, j}$ at some point $x(t) \in \mathcal{X}$ ) is measured, its structure is fixed once the structure of the underlying system is fixed. With additional assumptions or prior knowledge on the system, the structure of $R_{i, j}^{\perp}$ can be therefore assumed known and used to find the matrix $\mathcal{H}_{i, j} / R_{i, j}^{\perp}$. To illustrate this point consider the following example.

Example 2. Consider a nonlinear system described by equations of the form (1), with $x(t) \in \mathbb{R}, y(t) \in \mathbb{R}$, system mappings defined as

$$
f(x)=a_{1} x+a_{2} x^{2}, \quad h(x)=c x,
$$

for all $x \in \mathbb{R}, a_{1}, a_{2}, c \in \mathbb{R} \backslash\{0\}$, and initial condition $x(0)=x_{0} \in \mathbb{R} \backslash\{0\}$. The system satisfies the observability rank condition at any point of $\mathbb{R}^{3}$ except for the origin. Thus, the following considerations hold as long as the state of the system evolves away from the origin.

A direct computation shows that

$$
R_{2,3}=\left[\begin{array}{ccc}
0 & 0 & 0 \\
0 & 0 & 2 c a_{2}\left(a_{1} x+a_{2} x^{2}\right)^{2}
\end{array}\right],
$$

which, in turn, allows to obtain

$$
\Pi_{R_{2,3}}^{\perp}=\left[\begin{array}{ccc}
1 & 0 & 0 \\
0 & 1 & 0 \\
0 & 0 & 0
\end{array}\right] .
$$

Therefore, the system to be identified can be onedimensional and specified by mappings of the form (20) only if by multiplying the output matrix $Y_{2,3}$ by the projection matrix $\Pi_{R_{2,3}}^{\perp}$ derived above one obtains a rank one matrix $Y_{2,3} / R_{2,3}^{\perp}$. If that is the case, the system mappings $f$ and $h$ can be found with the procedure proposed in this section. 
Remark 3. If the system (1) is linear, i.e. its dynamics are described by equations of the form (5), equation (6) can be obtained by evaluating (16) along the trajectories of the system. Note that in the linear case the term $R_{i, j}$ is always zero, since $\left[f, \theta_{k}\right]=\left[A x, A^{k} x\right]=0$ for each non-negative integer $k$. It can be concluded that the former decomposition can be seen as a direct nonlinear counterpart of the latter and, to some extent, the remainder term $R_{i, j}$ can be interpreted as a measure of the nonlinearity of the system.

$\triangle$

Remark 4. The construction of the output matrix $Y_{i, j}$ requires the evaluation of a large number of high-order time derivatives of the input and the output signals. As discussed in the linear case, the time invariance property can be exploited to alleviate this issue in the linear case. Since the coefficient matrix $\Gamma_{i}$ is constant, the time invariance property allows to obtain a matrix equation formally identical to (6) with a reduced number of high-order time derivatives of the output signal. Although the class of autonomous nonlinear systems is also time-invariant, in general, it is not possible to adopt the same strategy to reduce the number of highorder time derivatives of the output signal. The reason for this is that the mapping $d \mathcal{H}_{i}$ depends explicitly on the state of the system.

\section{SUMmary AND FUtURE DIRECTIONS}

The problem of deterministic identification for autonomous linear and nonlinear systems has been studied. The main results of a version of discrete-time subspace identification for autonomous linear systems have been developed in continuous time. A novel identification framework for continuous-time autonomous nonlinear systems has been obtained by combining the subspace approach to linear identification and the differential-geometric approach to nonlinear control systems.

A number of questions and research directions are left open. Implementative aspects, such as effectively sampling the needed quantities or the use of timedelayed signals should be investigated. Finally, the significance for both theory and applications of the proposed nonlinear identification framework should be evaluated on real-world examples.

\section{REFERENCES}

[1] T. D. Söderström and P. G. Stoica, System identification. Prentice Hall, 1989.

[2] L. Ljung, System identification - Theory for the User (2nd edition). Prentice Hall, 1999.

[3] P. Van Overschee and B. De Moor, Subspace Identification for Linear System: Theory - Implementation - Applications. Kluwer Academic Publishers, 1996.

[4] T. Katayama, Subspace Methods for System Identification. Springer, 2006.

[5] M. Verhaegen and V. Verdult, Filtering and system identification: a least squares approach. Cambridge University Press, 2007.

[6] R. Johansson, M. Verhaegen, and C. T. Chou, "Stochastic theory of continuous-time state-space identification," IEEE Transactions on Signal Processing, vol. 47, no. 1, pp. 41-51, January 1999.
[7] T. Bastogne, H. Garnier, and P. Sibille, "A PMF-based subspace method for continuous-time model identification. Application to a multivariable winding process," International Journal of Control, vol. 74, no. 2, pp. 118-132, 2001.

[8] A. Ohsumi, K. Kameyama, and K. I. Yamaguchi, "Subspace identification for continuous-time stochastic systems via distribution-based approach," Automatica, vol. 38, no. 1, pp. 6379, 2002.

[9] V. Verdult, "Nonlinear System Identification: A State-Space Approach," Ph.D. dissertation, 2002.

[10] W. Favoreel, B. De Moor, and P. Van Overschee, "Subspace identification of bilinear systems subject to white inputs," IEEE Transactions on Automatic Control, vol. 44, no. 6, pp. 1157-1165, June 1999.

[11] H. Chen and J. M. Maciejowski, "A new subspace identification method for bilinear systems," University of Cambridge, Department of Engineering, Tech. Rep. CUED/F-INFENG/TR.357, 2000.

[12] D. Westwick and M. Verhaegen, "Identifying MIMO Wiener systems using subspace model identification methods ," Signal Processing, vol. 52, pp. 235 - 258, 1996.

[13] M. Lovera, T. Gustafsson, and M. Verhaegen, "Recursive subspace identification of linear and non-linear Wiener state-space models," Automatica, vol. 36, no. 11, pp. 1639-1650, 2000.

[14] J. C. Gómez and E. Baeyens, "Subspace identification of multivariable Hammerstein and Wiener models," in Proceedings of the 15th IFAC World Congress, Barcelona, Spain, 2002, pp. 418-418.

[15] I. Goethals, K. Pelckmans, J. A. K. Suykens, and B. De Moor, "Subspace identification of Hammerstein systems using least squares support vector machines," IEEE Transactions on Automatic Control, vol. 50, no. 10, pp. 1509-1519, October 2005.

[16] G. Kerschen, K. Worden, A. F. Vakakis, and J. C. Golinval, "Past, present and future of nonlinear system identification in structural dynamics," Mechanical Systems and Signal Processing, vol. 20, no. 3, pp. 505-592, 2006.

[17] S. Marchesiello and L. Garibaldi, "A time domain approach for identifying nonlinear vibrating structures by subspace methods," Mechanical Systems and Signal Processing, vol. 22, no. 1, pp. 81-101, 2008.

[18] V. Verdult, M. Verhaegen, and J. Scherpen, "Identification of nonlinear nonautonomous state space systems from inputoutput measurements," in Proceedings of IEEE International Conference on Industrial Technology, Goa, India, 2000, pp. 410-414.

[19] L. Ljung, "Perspectives on system identification," Annual Reviews in Control, vol. 34, no. 1, pp. 1-12, 2010.

[20] - "Approaches to identification of nonlinear systems," in Proceedings of the 29th Chinese Control Conference, Beijing, China, 2010, pp. 1-5.

[21] A. Isidori, Nonlinear Control Systems (3rd edition). Springer, 1995.

[22] H. Nijmeijer and A. Van der Schaft, Nonlinear dynamical control systems. Springer-Verlag, 1990.

[23] E. D. Sontag, Mathematical control theory: deterministic finite dimensional systems (2nd edition). Springer, 1998.

[24] H. Garnier, M. Mensler, and A. Richard, "Continuous-time model identification from sampled data: implementation issues and performance evaluation," International Journal of Control, vol. 76, no. 13, pp. 1337-1357, 2003.

[25] E. K. Larsson, M. Mossberg, and T. Söderström, "Estimation of continuous-time stochastic system parameters," in Identification of Continuous-time Models from Sampled Data. Springer, 2008, pp. 31-66.

[26] S. Diop, J. W. Grizzle, P. E. Moraal, and A. Stefanopoulou, "Interpolation and numerical differentiation for observer design," in Proceedings of the American Control Conference, Baltimore, MD, 1994, pp. 1329-1333.

[27] D. Serre, Matrices: Theory and Applications (2nd edition). Springer, 2010. 